\title{
Audiological characteristics in mucopolysaccharidosis: a systematic literature review
}

\author{
Flávia Teixeira Chimelo ${ }^{1}$ \\ https://orcid.org/0000-0002-8007-9745 \\ Liliane Aparecida Fagundes Silva ${ }^{1}$ \\ https://orcid.org/0000-0001-8985-0447
}

Chong Ae Kim²

https://orcid.org/0000-0002-1754-1300

Carla Gentile Matas ${ }^{1}$

https://orcid.org/0000-0001-5861-3342

\begin{abstract}
Universidade de São Paulo, Faculdade de Medicina FMUSP, Departamento de Fisioterapia, Fonoaudiologia e Terapia Ocupacional, São Paulo, São Paulo, Brasil.

Universidade de São Paulo, Faculdade de Medicina FMUSP, Unidade de Genética do Instituto da Criança do Hospital das Clínicas HCFMUSP, São Paulo, São Paulo, Brasil.
\end{abstract}

Research carried out at the Department of Physiotherapy, Speech-Language-Hearing Sciences and Occupational Therapy of the Medical School of the University of São Paulo - FMUSP - São Paulo, São Paulo, Brazil.

Conflict of interests: Nonexistent

\section{(c) (i)}

Received on: October 30, 2018 Accepted on: August 12, 2019

Corresponding address:

Flávia Teixeira Chimelo

Rua Cipotânea, 51, Cidade Universitária CEP: 05360-160 - São Paulo, São Paulo, Brasil

E-mail: fla_chimelo@hotmail.com

\section{ABSTRACT}

Purpose: to describe the audiological characteristics of patients with mucopolysaccharidosis.

Methods: after formulating the research question, three databases were considered for the search (Science Direct, Virtual Health Library and Web of Science); the following descriptors were used: mucopolysaccharidoses, hearing loss, and audiology. Articles were included that were original and complete, presenting audiological evaluation data in patients with mucopolysaccharidosis. Each study was classified according to the degree of recommendation and the level of scientific evidence, based on the criteria established by the Oxford Center for Evidence-based Medicine, and the results obtained from the evaluations were analyzed.

Results: of the 499 articles found, eight met the inclusion criteria. Pure tone audiometry and tympanometry were performed in all studies. The incidence of hearing loss in patients with MPS ranged from $54 \%$ to $100 \%$ of the cases, with conductive hearing loss corresponding to $30 \%$ and $58.33 \%$, mixed hearing loss corresponding to $28.5 \%$ and $66.66 \%$ and sensorineural hearing loss corresponding to less than $14 \%$. None of the studies described the responses of otoacoustic emissions and auditory evoked potentials.

Conclusion: the prevalence of hearing loss among individuals with MPS is higher than $54 \%$ of the cases, of which conductive and mixed are predominant.

Keywords: Hearing Loss; Mucopolysaccharidoses; Audiology 


\section{INTRODUCTION}

Mucopolysaccharidosis (MPS) belongs to a group of rare diseases, characterized by a deficiency of lysosomal enzymes, which are responsible for breaking the glycosaminoglycans (GAGs). The increasing lysosomal GAGs concentration results in serious growth deficits, skeletal deformities, hearing loss, poor joint mobility, grotesque facial characteristics, and organomegaly. The incidence for this group of rare diseases is considered to be from 1:10,000 to $1: 25,000^{1}$.

The MPSs may be classified in: I (Hurler, Hurler Scheie, and Scheie), II (Hunter), III (Sanfilippo), IV (Morquio), VI (Maroteaux-Lamy), and VII (Sly). Except for MPS II, a recessive X-linked inheritance, all other types of MPS are of autosomal recessive inheritance. In each type of MPS, there's a specific deficiency of different lysosomal enzymes; however, all the forms lead to a cell dysfunction triggered by the increasing accumulation of unbroken GAGs within the cells of various body tissues ${ }^{2,3}$.

In scientific literature, it's been registered that patients with MPS frequently present audiological alterations occasioned by the pathological GAGs deposition on the post-nasal space, the auditory tube, and the middle ear. Thus, these patients frequently need to be submitted to the insertion of ventilation tubes due to secretory or acute otitis media with effusion ${ }^{3,4}$. Conductive hearing loss is usually secondary to recurrent infections of the upper respiratory tract and serous otitis media, or to a deformity of the bony ossicles, wherefore the use of ventilation tubes is made necessary ${ }^{5}$.

Even though impairment of the middle ear is the more recurrent, the accumulation of GAGs may harm multiple regions of the auditory system, such as the cochlea, the auditory nerve and the brainstem, thus possibly resulting in sensorineural hearing loss, for whose treatment hearing aids may be a necessary clinical resource ${ }^{5}$.

Since such enzymatic deficiency has the potential to impair the integrity of the auditory system in many aspects, a broad knowledge of the audiological characteristics of these patients is made necessary, with the purpose of orienting the segments to be introduced in these individuals' clinical routine, as well as guiding new researches.

Hence, the objective of this study was to describe the audiological characteristics of patients with MPS.

\section{METHODS}

\section{Research strategy}

The first step of the research consisted in developing the question for the literature review: "What are the audiological characteristics of patients with mucopolysaccharidosis?" Making use of the PICO framework ${ }^{6}$, the research strategy was designed as follows:

- Patient: Individuals with MPS. Considering the rarity of the disease, no restriction was established regarding age group or MPS type;

- Intervention: Containing data of audiological assessment, including acoustic immittance, pure-tone audiometry, otoacoustic emission (OAE), and/or brainstem auditory evoked potential (BAEP);

- Comparison: Such studies were taken into consideration that compared their results to those of individuals without the disease (control group), or those that compared them to literature-defined normality criteria, or yet those without any kind of comparison;

- Outcomes: Either presenting or not audiological alterations, considering type, degree, configuration and/or prevalence of hearing loss in patients with MPS.

The systematic review of scientific literature was grounded on the search for studies published in the databases Science Direct, Virtual Health Library (VHL) and Web of Science. The following descriptors, found on Health Sciences Descriptors (DeCS, as abbreviated in Portuguese) and on Medical Subject Headings $(\mathrm{MeSH})$ both in English and in Portuguese, were taken into account: Hearing Loss, Mucopolysaccharidoses, and Audiology. The combination between descriptors was done with the Boolean operator "AND".

Thus, four searches were carried out between March and April 2018 on each database, using the following two combinations and their equivalent in Portuguese:

- "Mucopolysaccharidoses" AND "hearing loss";

- "Mucopolysaccharidoses" AND "audiology";

The strategies for searching, selecting and analyzing the articles followed the standards of the Preferred Reporting Items for Systematic Reviews (PRISMA) ${ }^{7}$.

\section{Selection criteria}

For this review, studies in accordance with the previously outlined PICO framework were considered, i.e., articles that had performed audiological assessment in patients with MPS, describing the findings in regard to type, degree and/or hearing loss configuration. 
Furthermore, concerning the search, national and international studies, published up until December 2017, in either Brazilian Portuguese or English, electronically available on the selected databases were taken into consideration.

Due to the small amount of articles related to the theme, the choice was for considering articles with levels $1,2,3$, and 4 , and with degrees of recommendation $\mathrm{A}, \mathrm{B}$ or $\mathrm{C}$, according to the Oxford Centre for Evidence-based Medicine ${ }^{8}$.

Expert's opinion pieces, congress summaries, book chapters and letters to the editor were excluded from this analysis.

\section{Data analysis}

In order to count the total number of articles found, a chart was filled in with the reference of each study approached. After carrying out the bibliographic survey in all databases with each descriptors combination, the next step was to research the articles to identify and exclude repeated titles.

In the sequence, two independent reviewers selected the articles that met the inclusion criteria, with the following elimination steps being kept in mind: reading of the titles; reading of the summaries; and, lastly, reading of the full texts. After completing each step, the reviewers conferred the selected articles; in the case of there being any divergence, it was solved by means of discussion between the reviewers.

After selecting the studies that met all the inclusion criteria, the articles were analyzed concerning important aspects to answer the research question in regard to objective, methodology and results obtained.

In relation to methodological analysis, each study was classified according to the degree of recommendation and level of scientific evidence, based on the criteria established by the Oxford Center for Evidencebased Medicine ${ }^{8}$.

The results were presented in accordance with the following aspects: time of publication, type of study, degree of recommendation and level of evidence, sample size, age group, procedures, type of MPS, classification of hearing loss and tympanometric curve, and main results found. It should be highlighted that, regarding longitudinal studies, the data from the last assessment were analyzed.

\section{LITERATURE REVIEW}

\section{Results from electronic databases}

The sum of research papers found in each search was of 499 articles; of all databases, the greatest amount proceeded from Science Direct. Making use of the descriptors in English, it became possible to find a greater amount of articles. After excluding the repeated titles, the total came to 453 published titles (Table 1).

Table 1. Description of the number of articles found according to descriptors and database

\begin{tabular}{lccc}
\hline \multirow{2}{*}{ Descriptor used } & \multicolumn{3}{c}{ Database } \\
\cline { 2 - 4 } & Science Direct & VHL & Web of science \\
\hline "Mucopolysaccharidoses" AND "hearing loss" & 423 & 37 & 15 \\
"Mucopolysaccharidoses" AND "audiology" & 22 & 0 & 0 \\
"Mucopolissacaridoses" AND "perda auditiva" & 2 & 15 & 0 \\
"Mucopolissacaridoses" AND "audiologia" & 0 & 0 & 0 \\
Total number of articles found & 447 & 52 & 0 \\
Number of articles on each database after excluding the repeated ones & 424 & 36 & 0 \\
\hline
\end{tabular}

\section{Final number of articles after excluding the repeated ones: $\mathbf{4 5 3}$}

Key: VHL - Virtual Health Library 
Following the inclusion criteria, the title of the 453 articles was read. After this step, 399 articles were excluded, and the summary of the remaining 54 was read. In the sequence, 15 articles were selected for full reading, resulting in eight articles that met all the criteria, which were then considered for analysis in this review.

The eight selected articles were analyzed in this review, and their main aspects were pointed out on Figure 2.

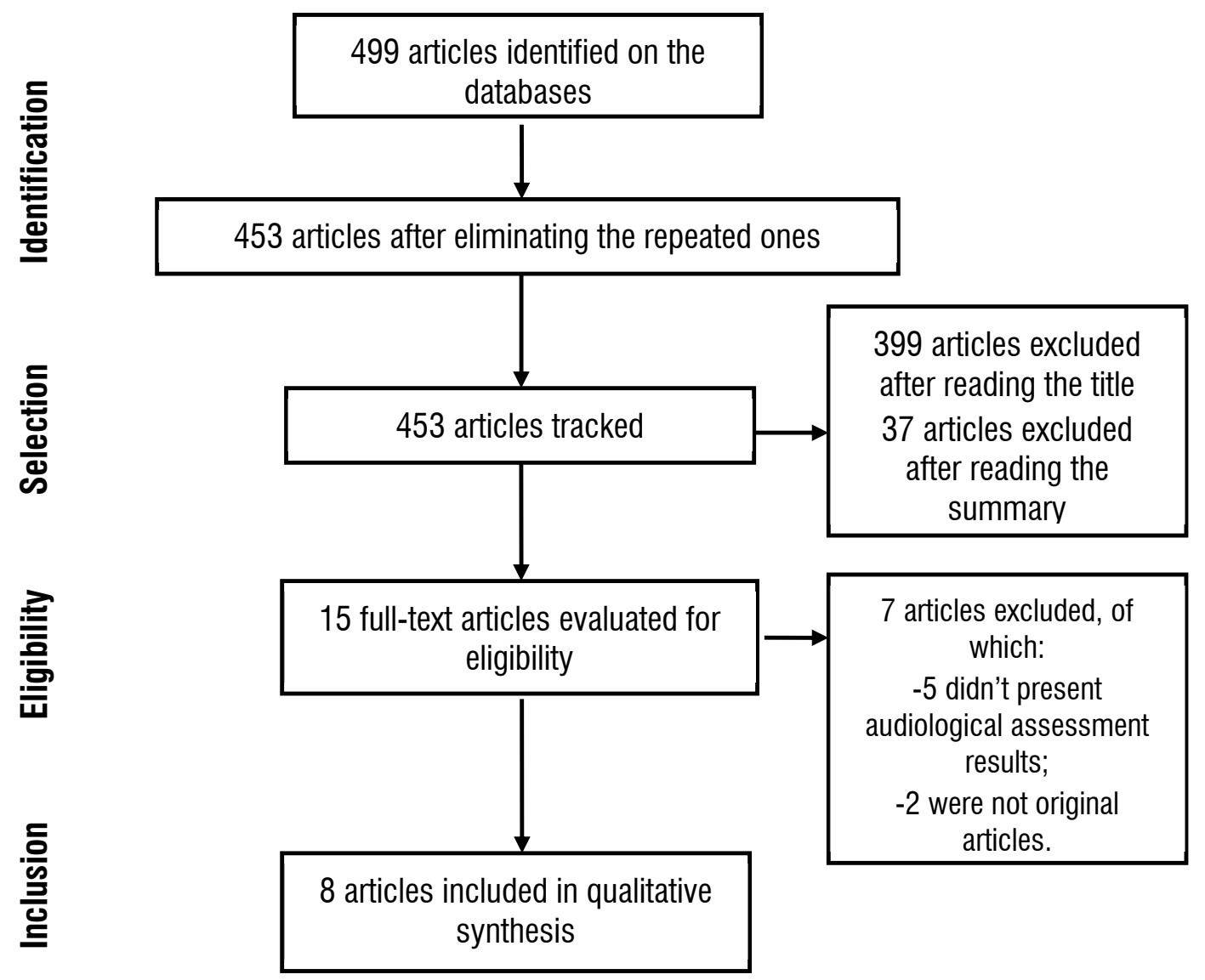

Figure 1. Flowchart of the selection of the articles, according to the model proposed by PRISMA 


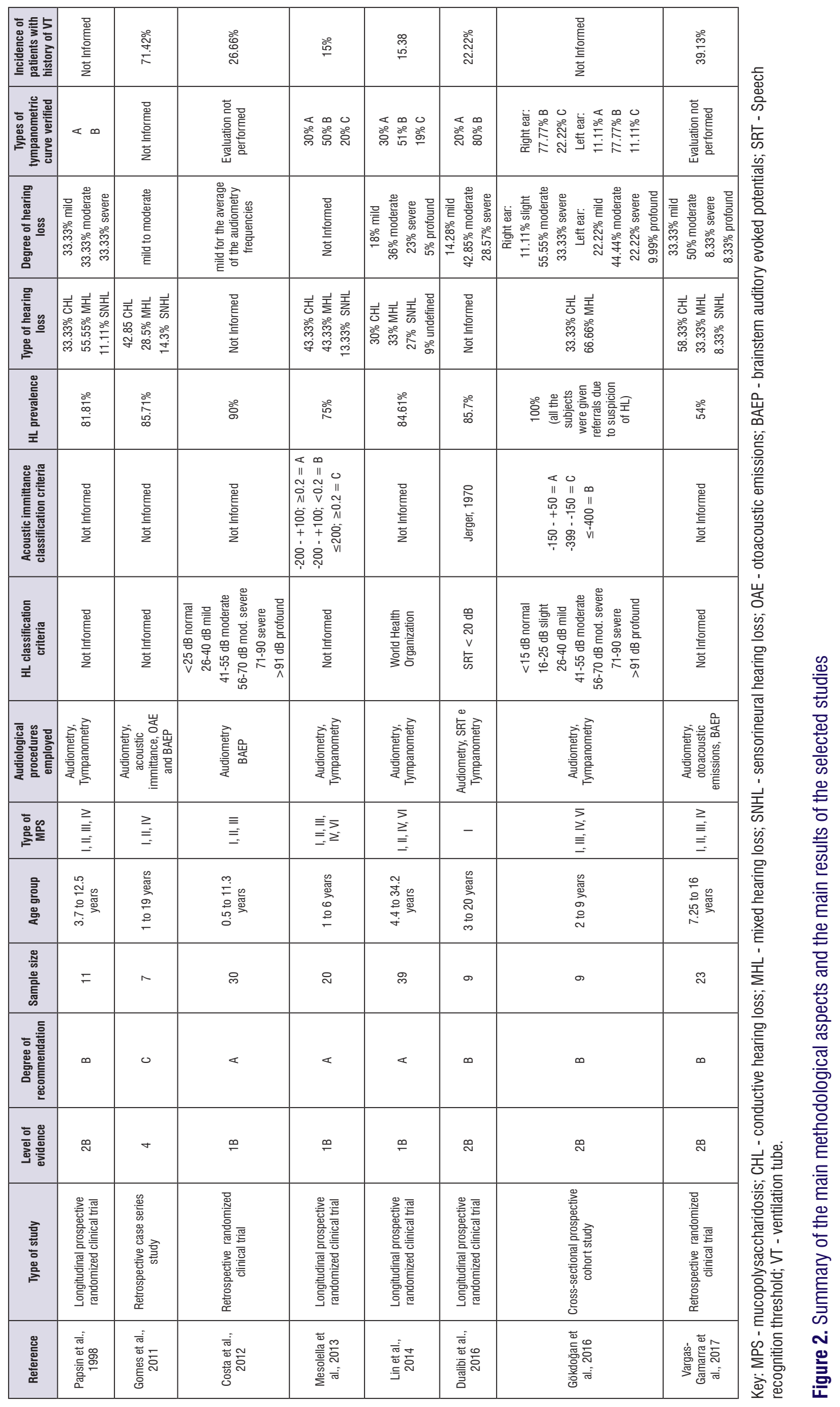




\section{Methodological characteristics}

The bibliographical survey brought to light that, even though there had been no restriction as to year of publication, of the studies containing auditory assessment in patients with MPS, only one had been published 20 years before ${ }^{9}$, while the other seven had been published less than seven years before ${ }^{10-17}$.

Regarding methodological aspects, it was noticed that there was a balance between types of study, three of them being retrospective $\mathrm{e}^{10-12}$ and five,

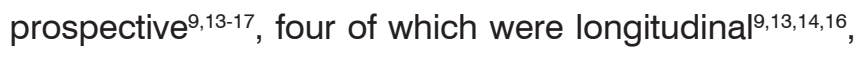
and one, cross-sectional ${ }^{15}$.

The age group was similarly broad, as the studies gathered patients between one and 34 years of age. Two articles observed less incidence and seriousness of hearing loss in younger individuals ${ }^{9,14}$; on the other hand, another study observed no correlation with age ${ }^{12}$. Attention is called to the fact that only three studies analyzed this variable, and with diverging results, so that little can be concluded, based on the studies included in this review, concerning the relation between age group and incidence of hearing loss in patients with MPS.

Still further on methodology, none of the studies had a control group for comparison. Hence, it wasn't possible to measure the prevalence of individuals with MPS in comparison to individuals without the disease. However, among the different types of MPS, one study pointed to the presence of more relevant hearing loss in individuals with MPS type I, II and IV ${ }^{9}$, while another study indicated greater incidence in individuals with MPS type I and $\mathrm{II}^{11}$. The authors described that it isn't possible yet to determine precisely the correlation between the type of MPS and hearing loss, and that more studies with a larger sample size are made necessary in order to confirm the findings.

As for the procedures, pure tone audiometry and tympanometry were the main clinical resources used for auditory assessment of the patients evaluated in the studies selected for this review. Only two studies included in its set of tests the otoacoustic emissions assessment ${ }^{10,11}$, and three evaluated the brainstem auditory evoked potentials ${ }^{10-12}$.

It's highlighted that not all the studies had clearly described the criteria adopted for the classification of normality values of auditory thresholds, as well as for the classification of the tympanometric curve, causing the findings to be obtained with less precision. Nonetheless, among the studies that did describe the reference standards, it was observed that the criteria followed the international recommendation standards, in which the normal values for auditory thresholds are inferior to $25 \mathrm{~dB} \mathrm{HL}$, and, regarding the type of tympanometric curve, the values are close to those proposed by Jerger, 1970, which is an internationally used reference.

\section{Audiological characteristics of patients with MPS}

In the acoustic immittance, although some studies found patients presenting type A tympanometric curve $^{9,13-16}$, it was observed that patients with MPS tend to present types $\mathrm{B}$ and $\mathrm{C}$ of tympanometric curve more often ${ }^{9,13-16}$. In the articles that furnished this information, these two types of tympanometric curve (B and $\mathrm{C}$ ) corresponded to more than $60 \%$ of all cases evaluated ${ }^{13-16}$.

This finding makes evident that damage to the middle ear can be pointed out as a frequent characteristic observed in individuals with MPS. In addition, it should be also emphasized that it wasn't possible to perform acoustic immittance in many patients of the studies, for many of them had a ventilation tube inserted in the tympanic membrane, due to the recurring otitis they presented. It was observed that between $15 \%^{13}$ and $71.42 \%{ }^{10}$ of the patients with MPS who took part of the selected studies had already been submitted to surgical interventions to insert the ventilation tube on the tympanic membrane.

Two of the analyzed studies didn't specify the type of hearing loss ${ }^{12,16}$. Of the six that described this variable, a great occurrence of conductive and mixed hearing loss was observed; the conductive hearing loss corresponded to about $30 \%{ }^{14}$ to $58.33 \%{ }^{11}$, and the mixed, to about $28.5 \%{ }^{10}$ to $66.66 \%{ }^{15}$. Regarding the sensorineural hearing loss, less incidence was observed, varying between $0 \%{ }^{15}$ and $14.3 \%{ }^{10}$. This finding highlights once again the great incidence of damage to the middle ear in patients with MPS; on the other hand, it makes evident that cochlear or neural alterations may also be found with less prevalence.

Furthermore, concerning the characteristics of the hearing loss in patients with MPS, of the five studies that described the incidence of degree of hearing loss $^{9,11,14-16}$, it was observed in general terms a greater incidence of moderate degree of hearing loss, corresponding to $33.33 \%{ }^{9}$ to $55.55 \%{ }^{15}$ of all losses.

None of the studies included in this review described the audiometric configuration; similarly, there's no description in the articles regarding what frequencies were affected the most. Taking this into account, it's 
important that future studies consider this analysis in order to better classify the audiological characteristics of this population.

In regard to the responses to the otoacoustic emissions, as well as to brainstem auditory evoked potentials, it was observed that these resources were included in the sets of audiological tests of the studies selected for this review only with the purpose of estimating or confirming the auditory thresholds, especially for the fact that some studies evaluated very young children, who weren't able to respond to behavioral assessments, yet. Thus, in these studies the characteristics of the variables analyzed in these procedures were not approached, such as response amplitude values for otoacoustic emissions, or wave latency and interpeak values for the brainstem auditory evoked potentials.

Attention should be called to the importance of more studies that include in their sets of audiological assessments the investigation of the integrity of the brainstem auditory pathway by means of analyzing wave latency and interpeak values for the auditory evoked potentials, since besides conductive damage, sensorineural damage was also observed in these patients ${ }^{10,12-15}$. In addition, it is known that the accumulation of GAGs may impair multiple regions of the auditory system, such as the cochlea, the auditory nerve and the brainstem ${ }^{17}$.

\section{Enzyme replacement therapy and stem cell transplant}

With the MPS metabolic disorder in view, it's currently available as a clinical resource the enzyme replacement therapy (ERT), which is a treatment consisting of periodical intravenous supply of the specific enzyme each patient is deficient of ${ }^{18}$. It's considered to be an effective therapeutic method, grounded on the periodical substitution of the defective enzyme that leads to more breaking of the GAGs on the tissues and organs, significantly improving some clinical characteristics ${ }^{19}$.

Of the studies selected for this review, one ${ }^{16}$ longitudinally evaluated the impact of the ERT on audiological and respiratory manifestations of patients with MPS. It was observed an improvement in the respiratory condition of the patients; however, improvement in hearing loss was not observed. According to the authors, other variables may have interfered on the audiological results, such as malformation of the ossicular chain, thickening of the middle ear mucous membrane, and tube dysfunction. Moreover, the authors highlighted that these patients' sensorineural hearing loss presents progressive character, tending to worsen even with the ERT, since the enzyme isn't capable of surpassing the hematoencephalic barrier ${ }^{16}$.

Although the abovementioned study has demonstrated such results, it's not possible to determine the impact of ERT on the hearing of individuals with MPS, yet. Another study which evaluated ERT mentioned that this therapy is still being investigated, and its efficacy has been proved only in regard to reducing non-neurological symptoms and pain ${ }^{11}$.

In another study, six patients with MPS II or VI showed improvement in auditory function after inserting ventilation tube and undergoing ERT for 1.9 to 8.5 years ${ }^{14}$.

Therefore, there's need for more studies with systematic hearing loss evaluation aiming at better clarifying the effects of ERT on these patients' hearing.

Moreover, regarding the clinical resources used for the improvement of the quality of life of patients with MPS, one of the studies evaluated the audiological profile before and after hematopoietic stem cell transplant, observing qualitative improvement in sensorineural hearing loss in $67 \%$ of the cases, which suggests that this method may improve these patients' hearing ${ }^{12}$.

Another study evaluated the effect of bone marrow transplant on the hearing of children with MPS, observing that the seriousness of hearing loss was less severe in the children who underwent such transplant than in those who weren't submitted to this porcedure9.

Based on all these data, the importance of speechlanguage-hearing and otorhinolaryngological evaluation and monitoring from the first months of life of patients with MPS is emphasized, since the earliest possible medical follow-up and intervention may avoid the aggravation of the otologic condition and enable improved hearing conditions, thus favoring the development of oral language and improving the quality of life of these individuals.

\section{CONCLUSION}

Based on articles surveyed for this review, it can be concluded that the prevalence of hearing loss in individuals with MPS is superior to $54 \%$ of the cases, of which the most predominant are the conductive and the mixed of moderate degree. Considering the high incidence of damage to the middle ear, the insertion of ventilation tube is a resource used in most of the cases, 
due to the recurring conditions of secretory or acute otitis media with effusion.

\section{REFERENCES}

1. Yeung AH, Cowan MJ, Horn B, Rosbe KW. Airway management in children with mucopolysaccharidoses. Arch Otolaryngol Head Neck Surg. 2009;135(1):73-9.

2. Neufeld EF, Muenzer J. The mucopolysacharidoses. In: Scriver CR, Beaudet AL, Sly WS, Valle D (orgs). The metabolic and molecular bases of inherited diseases. 8th ed. New York: McGray-Hill; 2001.p.3421-52.

3. Simmons MA, Bruce IA, Penney S, Wraith E, Rothera MP. Otorhinolaryngological manifestations of the mucopolysaccharidoses. Int. J. Pediatri. Otorhinolaryngol. 2005;69(5):589-95.

4. Bicalho CG, Rezende MM, Nogueira AMCM, Paulon RMC, Acosta AX. A importância da avaliação otorrinolaringológica de pacientes com mucopolissacaridose. Arquivos Int. Otorrinolaringol. 2011;15(3):290-4 .

5. Schleier E, Streubel HG. Aspectos foniátricos de crianças com Mucopolissacaridose. Folia. Phoniatr. 1976;28:65-72.

6. Santos CMC, Pimenta CAM, Nobre MRC. The PICO strategy for the research question construction and evidence search. Rev Lat Am Enfermagem. 2007;15(3):508-11.

7. Moher D, Liberati A, Tetzlaff J, Altman DG. PRISMA Group. Preferred reporting items for systematic reviews and meta-analyses: the PRISMA Statement. Ann Intern Med. 2009:18;151(4):264-9.

8. Oxford Centre for Evidence-based Medicine Levels of Evidence. Março de 2009. Disponível em: http://www.cebm.net/oxford-centre-evidencebased-medicine-levels-evidence-march-2009/. Acesso em: 25 de Junho de 2018.

9. Papsin BC, Vellodi A, Bailey CM, Ratcliffe PC, Leighton SE. Otologic and laryngologic manifestations of mucopolysaccharidoses after bone marrow transplantation. Otolaryngol Head Neck Surg. 1998;118(1):30-6.

10. Gomes AB, Pereira RG, Vassoler TMF, Alcantara LJL, Zimmermann E, Santos MLSF. Avaliação audiológica de pacientes com mucopolissacaridose em um hospital pediátrico. Arquivos Int. Otorrinolaringol. 2011;15(2):203-7.

11. Vargas-Gamarra MF, de Paula-Vernetta C, Vitoria Miñana I, Ibañez-Alcañiz I, Cavallé-Garrido L, Alamar-Velazquez A. Audiological findings in children with mucopolysaccharidoses type i-iv. Acta Otorrinolaringologica. 2017;68(5):262-8.

12. Costa V, O'Grady G, Jackson L, Kaylie D, Raynor E. Improvements in sensorineural hearing loss after cord blood transplant in patients with mucopolysaccharidosis. Arch Otolaryngol Head Neck Surg. 2012;138(11):1071-6.

13. Mesolella M, Cimmino M, Cantone E, Marino A, Cozzolino M, Della Casa $R$ et al. Management of otolaryngological manifestations in mucopolysaccharidoses: our experience. Acta Otorhinolaryngologica Italica. 2013;33(4):267-2.

14. Lin HY, Shih SC, Chuang CK, Lee KS, Chen MR, Lin $\mathrm{HC}$ et al. Assessment of hearing loss by pure-tone audiometry in patients with mucopolysaccharidoses. Mol Genet Metab. 2014;111(4):533-8.

15. Gökdoğan Ç, Altinyay Ş, Gökdoğan O, Tutar $H$, Gündüz B, Okur İ et al. Audiologic evaluations of children with mucopolysaccharidosis. Braz J Otorhinolaryngol. 2016;82(3):281-4.

16. Dualibi APFF, Martins AM, Moreira GA, Azevedo MF, Fujita RR, Pignatari SSN. The impact of laronidase treatment in otolaryngological manifestations of patients with mucopolysaccharidosis. Braz. j. Otorhinolaryngol. 2016;82(5):522-8.

17. Mustacchi Z, Peres S. Genética bioquímica Erros inatos do matabolismo. In: Mustacchi Z, Peres $S$ (orgs). Genética baseada em evidências: Síndromes e heranças. São Paulo: CID editora; 2000. p. 429-77.

18. Barton NW, Furbish FS, Murray GJ, Garfield M, Brady RO. Therapeutic response to intravenous infusions of glucocerebrosidase in a patient with Gaucher disease. Proc Nat Acad Sci USA. 1990;87(5):1913-6.

19. Viana GM, Cavaleiro R, Alves E, Souza IC, Feio $\mathrm{R}$, Leistner-Segal $\mathrm{S}$ et al. Mucopolissacaridoses no norte do Brasil: rastreamento de mutações direcionadas e excreção de glicosaminoglicanos urinários em pacientes submetidos à terapia de reposição enzimática. Genet Mol Biol. 2011;34(3):4105. 